\title{
Ultrasonic based concrete defects identification via wavelet packet transform and GA-BP neural network
}

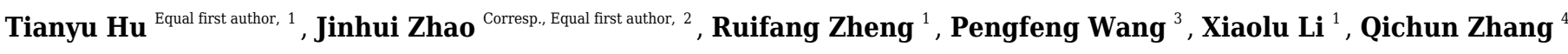 \\ ${ }^{1}$ College of Mechanical and Electrical Engineering, China Jiliang University, Hangzhou, China \\ 2 Key laboratory for technology in rural water management of Zhejiang province, College of Electrical Engineering,, Zhejiang University of Water \\ Resources and Electric Power, Hangzhou, China \\ ${ }^{3}$ College of Modern Science and Technology, China Jiliang Univercity, Hangzhou, China \\ 4 Department of Computer Science, University of Bradford, Bradford, United Kingdom \\ Corresponding Author: Jinhui Zhao \\ Email address: jhzhao2009@zju.edu.cn
}

Concrete is the main material in building. Since its poor structural integrity may cause accidents, it is significant to detect defects in concrete. However, it is a challenging topic as the unevenness of concrete would lead to the complex dynamics with uncertainties in the ultrasonic diagnosis of defects. Note that the detection results mainly depend on the direct parameters, e.g. the time of travel through the concrete. The current diagnosis accuracy and intelligence level are difficult to meet the design requirement for automatic and increasingly high-performance demands. To solve the mentioned problems, our contribution of this paper can be summarized as establishing a diagnosis model based on the GA-BPNN method and ultrasonic information extracted that helps engineers identify concrete defects. Potentially, the application of this model helps to improve the working efficiency, diagnostic accuracy and automation level of ultrasonic testing instruments. In particular, we propose a simple and effective signal recognition method for small-size concrete hole defects. This method can be divided into two parts: 1) signal effective information extraction based on wavelet packet transform (WPT), where mean value, standard deviation, kurtosis coefficient, skewness coefficient and energy ratio are utilized as features to characterize the detection signals based on the analysis of the main frequency node of the signals, and 2) defect signal recognition based on GA optimized back propagation neural network (GA-BPNN), where the cross-validation method has been used for the stochastic division of the signal dataset and it leads to the BPNN recognition model with small bias. Finally, we implement this method on 150 detection signal data which are obtained by the ultrasonic testing system with $50 \mathrm{kHz}$ working frequency. The experimental test block is a C30 class concrete block with $5 \mathrm{~mm}, 7 \mathrm{~mm}$, and $9 \mathrm{~mm}$ penetrating holes. The information of the experimental environment, algorithmic parameters setting and signal processing procedure are described in detail. The average 
recognition accuracy is $91.33 \%$ for the identification of small size concrete defects according to experimental results, which verifies the feasibility and efficiency. 
1 Ultrasonic based Concrete Defects Identification via

2 Wavelet Packet Transform and GA-BP Neural Network

3

\section{Tianyu Hu ${ }^{*}, 1$, Jinhui Zhao ${ }^{*}, 2$, Ruifang Zheng ${ }^{1}$, Pengfeng Wang ${ }^{3}$, Xiaolu $\mathrm{Li}^{1}$ and Qichun Zhang ${ }^{4}$}

${ }^{1}$ College of Mechanical and Electrical Engineering, China Jiliang University, Hangzhou, Zhejiang Province, China

${ }^{2}$ Key laboratory for technology in rural water management of Zhejiang province, College of Electrical Engineering, Zhejiang University of Water Resources and Electric Power, Hangzhou Zhejiang Province, China

${ }^{3}$ College of Modern Science and Technology, China Jiliang University, Hangzhou, Zhejiang Province, China

${ }^{4}$ Department of Computer Science, University of Bradford, Bradford, BD7 1DP, the United Kingdom

* These authors contributed to the work equally and should be regarded as co-first authors. Corresponding Author: Jinhui Zhao

No. 583, Xuelin Street, Jianggan District, Hangzhou, Zhejiang Province 310018, China Email address: jhzhao2009@zju.edu.cn

\section{ABSTRACT}

Concrete is the main material in building. Since its poor structural integrity may cause accidents, it is significant to detect defects in concrete. However, it is a challenging topic as the unevenness of concrete would lead to the complex dynamics with uncertainties in the ultrasonic diagnosis of defects. Note that the detection results mainly depend on the direct parameters, e.g. the time of travel through the concrete. The current diagnosis accuracy and intelligence level are difficult to meet the design requirement for automatic and increasingly high-performance demands. To solve the mentioned problems, our contribution of this paper can be summarized as establishing a diagnosis model based on the GA-BPNN method and ultrasonic information extracted that helps engineers identify concrete defects. Potentially, the application of this model helps to improve the working efficiency, diagnostic accuracy and automation level of ultrasonic testing instruments. In particular, we propose a simple and effective signal recognition method for small-size concrete hole defects. This method can be divided into two parts: 1) signal effective information extraction based on wavelet packet transform (WPT), where mean value, standard deviation, kurtosis coefficient, skewness coefficient and energy ratio are utilized as features to characterize the detection signals based on the analysis of the main frequency node of the signals, and 2) defect signal recognition based on GA optimized back propagation neural network (GA-BPNN), where the cross-validation method has been used for the stochastic division of the signal dataset and it 
40 leads to the BPNN recognition model with small bias. Finally, we implement this method on 150

41 detection signal data which are obtained by the ultrasonic testing system with $50 \mathrm{kHz}$ working 42 frequency. The experimental test block is a C30 class concrete block with $5 \mathrm{~mm}, 7 \mathrm{~mm}$, and $9 \mathrm{~mm}$ 43 penetrating holes. The information of the experimental environment, algorithmic parameters 44 setting and signal processing procedure are described in detail. The average recognition accuracy 45 is $91.33 \%$ for the identification of small size concrete defects according to experimental results, 46 which verifies the feasibility and efficiency.

\section{INTRODUCTION}

48 Concrete materials are widely used in modern buildings. It is a non-uniform material mixed with 49 cement, sand, gravel and water. The random distributions of coarse aggregate and cement mortar 50 are the causes of the heterogeneity of concrete. There exist various forms of deterioration and 51 defects of concrete structures because of aging and environmental damage, such as internal voids 52 (Bien, Kaminski \& Kuzawa,2019). Among the main health problems of concrete, surface defects are relatively easy to be detected. However, internal defects are hidden in the concrete, which is difficult to detect and is more harmful. It is significant to detect and analyze the internal defects of concrete structures in time to avoid the potential related accidents.

Commonly used methods of non-destructive testing include electromagnetic, radiological and ultrasound (Schabowicz, 2019). Ultrasonic has the advantages of strong penetrating power and high sensitivity, so it is mostly used in material defect detection (Janku, et al., 2019). In actual inspection tasks, ultrasonic detection of concrete defects is based on the observation of acoustic parameters, propagation time, amplitude and main frequency of ultrasonic detection signals, etc. (Ushakov, Davydov, 2013; Ozsoy, Koyunlu \& Ugweje, 2017). For example, NDT James V-C-400 V-Meter MK IV still uses the ultrasonic pulse velocity method to characterize the detection signal. These applied methods are susceptible to individual subjective factors and experience levels. The applications of modern signal processing and artificial intelligence algorithms can achieve the automatic recognition of signals and also improve recognition efficiency and accuracy (TibaduizaBurgos \& Torres-Arredondo, 2015; Javed et al., 2020). It is necessary to obtain effective information to characterize different types of signals before performing detection signal recognition. At present, many methods have been used to find the effective features of complex signals (Cheema \& Singh, 2019). These signal analysis methods are mainly sparse representation, Hilbert-Huang transform, Fourier transform, wavelet transform, and so on (Liu et al., 2021; Kumar \& Kumar, 2019; Bochud et al., 2015; Rodriguesa et al., 2019). However, it is difficult to extract the key information from concrete ultrasonic detection signals due to the noise influence and many mutational components, such as mutational amplitude (Cheema \& Singh 2019; Combet, Gelman, \& LaPayne, 2012). Among these signal preprocessing methods, wavelet transform can effectively deal with the non-stationary and high-noise complex signals. This method has been applied to process ultrasonic signals (Acciani et al.,2010). Machine learning models are established with simple structures which are suitable for small sample dataset, while the scholars often choose these methods to identify detection signals (Iyer et al., 2012). For now, commonly used machine learning algorithms include support vector 
80

81

82

83

84

85

86

87

88

89

90

91

92

93

94

95

96

97

98

99

100

101

102

103

104

105

106

107

108

109

110

111

112

113

114

115

116

117

118

119

machine, neural network, etc. (Rajput et al., 2021; Xu \& Jin, 2018). As a class of neural networks, BP neural network (BPNN) is a classic model. It has strong nonlinear mapping ability and simple structure (Wang, 2015). After optimization by genetic algorithm, the fitting ability and running speed can be improved. Note that BPNN is widely used in the field of pattern recognition, where deep learning is one of the most popular methods in pattern recognition. In the field of ultrasonic inspection, deep learning has been used to identify inspection images (Slonski, Schabowicz, \& Krawczyk, 2020). However, deep learning was rarely used to recognize concrete ultrasonic detection signals due to the high hardware performance requirements (Shrestha \& Mahmood, 2019).

Saechai S. et al. (Saechai, Kongprawechnon \& Sahamitmongkol, 2012) used the support vector machine to identify the defect detection signals of concrete which obtained higher recognition accuracy. Chen Y. et al. (Chen \& Ma, 2011) extracted features of the weld detection signal by wavelet packet transform and used radial basis function neural network to recognize defects. Zhang K.X. et al. (Zhang et al., 2020) used genetic algorithm-back propagation neural network to evaluate the laser ultrasonic fault signals of uniform metal structures. The composition of the concrete selected in our paper is more complex than the research objects in the literatures. When these methods are used directly to identify concrete detection signals, the performance would be deteriorated. Therefore, a novel ultrasonic-based solution should be developed for concrete defect detection.

In this paper, we propose an intelligent method to process the ultrasonic lateral detection signals of penetrating holes in concrete. The main contributions and objective are summarized as follows:

- To improve the performance of more effective calculation and high identification accuracy, the ultrasonic detection signals are decomposed by WPT in order to extract the useful information in the detection signal. As a result, we extract the 5 effective features of the processed signal.

- Genetic algorithm has been used to optimize the structural parameters of the BP neural network. In the experiments with measured data, the average classification accuracy of GABPNN is increased by $4.66 \%, 4 \%$, and $5.33 \%$ compared with BPNN, SVM, and RBF, respectively.

- This paper presents a generalized research framework on the processing and recognition of concrete ultrasonic detection signals, which lays the technical foundation for achieving the intelligent and automatic detection of concrete.

The rest of the paper has been organized as follows: In section 2, we describe the whole algorithmic procedure and principles briefly. And we present the experimental system and algorithmic parameters setting in the 3 rd section. The test results and analysis are presented in the 4th section. We draw a conclusion in the 5 th section.

\section{THE PROCESS OF THE PROPOSED ALGORITHM}

The ultrasonic pulse velocity (UPV) method is widely used in ultrasonic testing instruments which cannot meet the needs of small-size concrete defect detection. The levels of intelligence and automation of concrete testing instruments need to be improved urgently. To solve this problem, 
120

121

122

123

124

125

126

127

128

129

130

131

132

133

134

135

136

137

138

139

140

141

142

we propose a method based on WPT and GA-BPNN. In particular, the presented algorithm in this paper consists of three parts. First, wavelet packet transform is used to attenuate noise and retain effective information from the non-stationary concrete ultrasonic detection signals. Then, the features of processed signals are extracted as the feature vector. Finally, we use the BPNN optimized by the improved GA to identify the detection signals and the K-fold cross-validation is introduced to verify the stability and generalization of GA-BPNN. We describe the main steps in the following subsections.

\section{Wavelet packet transform}

Wavelet transform is a multi-resolution analysis method (Babouri et al., 2019), which is a process of using wavelet basis functions to decompose a signal into components of different frequency bands, processing the wavelet packet coefficients, and reconstructing these components into a complete signal (Kim et al., 2020). When using the wavelet transform to process a non-stationary signal, there are different resolutions at different locations. WPT can accurately obtain the high and low-frequency components of the signal (Schimmack \& Mercorelli, 2018). Therefore, WPT can be considered as an effective pre-processing algorithm for feature extraction. However, the wavelet transform cannot extract the detailed information of detection signals.

\section{Decomposition and reconstruction of WPT}

The structure diagram of the three-layer decomposition of wavelet packet is given in Fig. 1.

In Fig. 1, $S$ means an original signal. Then, $S$ can be decomposed according to the equation (1) to obtain $A$ and $D$. $A$ is a low-frequency component and $D$ is a high-frequency component after each decomposition of an original signal. Continuously, we decompose $A$ and $D$ in the same way. Finally, $S$ is decomposed into eight components at different frequency bands.

The basic calculation formulas of WPT are shown in equations (1-2).

$$
\begin{aligned}
& \left\{\begin{array}{l}
d_{j}^{2 n}[k]=\sum_{l \in Z} h_{l-2 k} d_{j+1}^{n}[l] \\
d_{j}^{2 n+1}[k]=\sum_{l \in Z} g_{l-2 k} d_{j+1}^{n}[l]
\end{array}\right. \\
& d_{j}^{2 n+1}[k]=\sum_{l \in Z} h_{k-2 l} d_{j}^{2 n}[l]+\sum_{l \in Z} g_{k-2 l} d_{j}^{2 n+1}[l]
\end{aligned}
$$

143 where $d_{j+1}^{n}$ represents a wavelet packet coefficient sequence of the signal to be decomposed, $j$ represents a scale factor, $n$ represents the number of frequency bands, $k$ and $l$ are the positions of coefficients sequences, $d_{j}^{2 n}$ and $d_{j}^{2 n+1}$ represent wavelet packet coefficients sequences of signals after decomposition, $\boldsymbol{h}$ represents orthogonal real coefficients matrices of low-pass filters, and $\boldsymbol{g}$ represents orthogonal real coefficients matrices of high-pass filters.

148 We use the cost function to select the wavelet packet basis for the signal decomposition. At present, 149 the Shannon entropy (Shi et al., 2021) is widely used where the entropy of the wavelet packet 150 coefficient sequence $\boldsymbol{d}=\left\{d_{j}\right\}$ is defined by equation (3).

$$
M(d)=-\sum_{j} P_{j} \log _{2} P_{j}
$$

151 where $P_{j}=\frac{\left|d_{j}\right|^{2}}{\|d\|^{2}}$, and when $P=0, P \log _{2} P=0$. 


\section{Wavelet basis function selection}

153 How to choose the appropriate wavelet basis function is vital besides WPT decomposes ultrasonic 154 detection signals precisely. Samaratunga D. et al. (Samaratunga, Jha \& Gopalakrishnan, 2016) 155 think the time-frequency change of non-stationary signal is well represented by the Daubechies 156 wavelet function in the time-frequency domain. In this paper, the db15 wavelet is selected as the 157 base function of WPT according to the decomposition experiment analysis.

158 Ultrasonic signal features selection

159 In pattern recognition, feature extraction is normally used for two processes: object feature data 160 collection and classification. The quality and property of feature data greatly affect the design and 161 the performance of pattern recognition classifiers, e.g., monotonicity, which is a key problem of 162 pattern recognition.

163 Scholars used wavelet coefficients after wavelet transform as feature vectors, which resulted in the

164

165

166

167

168

169

170

171

172

173

174

175

176

177

178

179

180

181

182

183

184

185

186

187

188

189

190

191 very high-dimensional input data of the recognition model (Cruz et al., 2016). Wan P. et al. (Wan $\& \mathrm{Li}, 2014$ ) only extracted one type of feature including the energy ratio of each node after wavelet packet decomposition as the feature vector of identifying defects in carbon-fiber-reinforced polymer. Wang X.K. et al. (Wang et al., 2019) selected 9 features including the average peak spacing, dominant frequency, etc., to identify weld quality defects. Furthermore, scholars also choose features such as mean value, standard deviation, kurtosis, etc. as the inputs of ultrasonic detection signal recognition models (Virmani et al, 2017).

Based on commonly used features in the field of ultrasonic testing, we have selected useful and non-redundant features by analyzing the calculation formulas of the features and conducting experimental tests. For example, the calculation formulas and physical meaning of mean square value and energy are very similar, and they are not used as features collectively. Finally, the five features of mean value, standard deviation, kurtosis coefficient, skewness coefficient and energy ratio are retained (Wan \& Li, 2014; Zhang, Duffy \& Orlandi, 2017).

In order to make the feature values in the same order of magnitude and improve the convergence speed of the model, we normalize the extracted features (Bagan et al., 2009). The five feature values are mapped to $[-1,1]$ and the normalized feature values are taken as input variables of the BPNN model in this paper.

\section{GA-BP neural network (GA-BPNN)}

\section{BP Neural Network (BPNN)}

A BPNN is made up of an input layer, a hidden layer, and an output layer. The input signal of BPNN propagates forward, and the error propagates backward. It can approximate the function of finite discontinuities (Wang, 2015). In addition, it has a powerful ability to deal with nonlinear problems. The structure is shown in Fig. 2.

In Fig. 2, $\boldsymbol{x}$ is input data, $\omega_{\mathrm{ij}}$ is the weight between the input layer $i$ and the hidden layer $j, \boldsymbol{\alpha}$ is biases of the hidden layer, $f_{1}$ is activation functions used for the hidden layer, $\omega_{\mathrm{jk}}$ is the weight between the hidden layer $j$ and the output layer $k, \boldsymbol{\beta}$ is biases of the output layer, $f_{2}$ is the activation function used for the output layer, and $\boldsymbol{y}$ is the output of the network.

\section{Genetic algorithm (GA)}


192 The outputs of the BPNN are calculated according to its input-output function built on the

193

194

195

196

197

198

199

200

201

202

203

204

205

206

207

208

209

210

211

212

213

214

215

216

217

218

219

220

221

222

223

224

225

226

227

228

229

230

231

generated weights, biases and number of hidden nodes. In this paper, the improved GA (Peng et al., 2013) has been used to optimize initial weights, biases and the number of hidden layer nodes. This method can make BPNN convergent fast with higher precision (Han \& Huang, 2019). According to the description of the improved GA (Peng et al., 2013), we use binary to code variables of the number of hidden layer node and use real numbers to code variables of the corresponding weights and biases for building candidate solutions in GA. We assume the maximum number of hidden layer node in the BPNN is $l$, and the number of input and output layer nodes in the network are $n$ and $m$, respectively. Then the total number of optimization variables is $1+(l \cdot n)+l+(l \cdot m)+m$. The coding of all parameters in a candidate solution is shown in Fig. 3. For instance, if the number of hidden layer nodes is represented by a q-bit 0 to 1 string, the range of the number of hidden layer node is 0 to $2^{\mathrm{q}}-1$, and $l=2^{\mathrm{q}}-1$.

In Fig. 3, $q$ is the number of candidates hidden layer node, $l$ is the maximum number of hidden layer node, $0<q \leqslant l, n$ is the number of nodes in the input layer, $m$ is the number of nodes in the output layer. Furthermore, errors between the actual values and the output values of the BPNN are calculated, then the reciprocal of the sum of squared errors is used as the fitness function in the GA.

In this paper, the roulette wheel method is used as the selection operator. Two individuals are selected by the selection operator. Then we use the one-point crossover method to process the binary coding arrays. The arithmetic crossover operator is used for the real number encoding sequences. For binary coding arrays, the simple mutation operator is used. We apply the nonuniform mutation operator to the real coding sequences in this paper. Briefly, we first encode the variables that need to be optimized; next, the fitness values in the initial population are calculated; then, we perform selection, crossover, and mutation operations to generate a new generation of population and obtain the maximum fitness value of each generation; finally, the optimal variable values are obtained by decoding the individual with the largest fitness value among all offspring.

\section{Overall steps of the WPT and GA-BPNN method}

To describe the proposed concrete ultrasonic detection signal identification method, the main steps are summarized as follows.

Step1: The ultrasonic detection signal is decomposed into three layers by WPT sub-algorithm, and the wavelet packet coefficients in the main frequency node are extracted to reconstruct the signal; Step2: The five feature variables of the reconstructed signals are calculated to establish the feature dataset;

Step3: Adopt K-fold cross-verification method to divide the dataset;

Step4: The genetic algorithm is executed to calculate the optimal configuration parameter of BPNN; select the optimal parameters of BPNN from the optimal solutions of GA, then obtain an optimized BPNN;

Step5: Use the test dataset to test the BPNN, output the recognition results.

The flowchart of the proposed method is given in Fig.4.

\section{EXPERIMENTAL ENVIRONMENT AND TEST}

Peer) Comput. Sci. reviewing PDF | (CS-2021:04:60010:1:1:NEW 9 Jun 2021) 


\section{Experimental set-up and dataset}

233 As an engineering application, we apply the ultrasonic transmission detection method to the 234 practical ultrasonic detection system in which we use the P28F ultrasonic probes with the $50 \mathrm{kHz}$ 235 working frequency to generate the ultrasonic signals. An \pm 80 -Volt square wave pulse signal is 236 generated at the transmitting end to excite the ultrasonic probe vibration. The signal sampling 237 frequency of the receiving end is $1 \mathrm{MHz}$. The analog-to-digital conversion module used for data 238 acquisition is 12 bits. Each detection signal used in this paper contains a total of 18,000 sampling 239 points in 6 cycles. The Fig. 6 is concrete test sample. Concrete is mainly composed of cement, 240 sand, coarse aggregate and water, the material ratio of C30 class concrete is 461, 175, 512 and $2411252 \mathrm{~kg} / \mathrm{m}^{3}$. The size of the sample block is pre-specified as follows: the length is $30 \mathrm{~cm}$, the width 242 is $20 \mathrm{~cm}$ and the height is $20 \mathrm{~cm}$.

243 The experimental data are obtained by sampling repeatedly at the different positions shown in Fig. 244 7. The white dots of test points shown in Fig. 7 are all measured evenly. Hole defects are available 245 in three sizes. The distance between two penetrating holes is $85 \mathrm{~mm}$. The diameters of penetrating 246 holes are $5 \mathrm{~mm}, 7 \mathrm{~mm}$ and $9 \mathrm{~mm}$, respectively. Three test points are placed on the surface of each 247 kind of hole defect. Six test points of the defect-free structure are located between the points over 248 the holes. The horizontal and vertical distances between the detection positions are both $45 \mathrm{~mm}$. 249 Fifteen detection positions are arranged on the concrete surface, and 10 detection data signals are 250 obtained for each detection point. In this case study, a total of 150 ultrasonic transmission detection 251 data samples are obtained through the experimental device in Fig. 8, including 60 sample data 252 signals from the non-defective structure, and 90 sample data signals from the defective structure. 253 Fig. 5 shows the experimental data acquisition process of the detection system.

\section{Algorithm parameter settings}

255 According to the wavelet basis function selection rule and the distribution of these detection 256 signals' time-frequency characteristics, the db15 wavelet function is selected to perform the three257 layer WPT. And most of the valid information of the signal is included in the first node of the third 258 layer after decomposing the detection signals.

259 In algorithm experiments, our computer is 64-bit Windows operation system. The hardware 260 configuration includes $2.08 \mathrm{GHz} \mathrm{CPU}$, Inter Core i5-8400 with 6 cores, and $32 \mathrm{~GB} 2400 \mathrm{MHz}$ 261 DDR4 memory. The application software is MATLAB R2014a version. The main parameter 262 setting of the proposed algorithm is given as follows.

263 The WPT parameters setting is: the wavelet basis function is $\mathrm{db} 15$, the number of decomposition 264 levels is 3, and the optimal wavelet basis Shannon entropy is selected.

265 The GA algorithmic parameters setting is: the maximum genetic algebra $g$ is 100 , the population 266 size $p$ is 50, the binary code length $q$ is 5 , the crossover probability $P c$ is 0.7 , and the mutation 267 probability $P m$ is 0.05 .

268 The BPNN algorithmic parameters setting is: the number of input nodes is 5, the number of output 269 nodes is 2 , the training stop condition is that the model error reaches 0.001 or the epochs of training 270 reaches 1000 , and the learning rate is 0.01 . Simultaneously, the cross-validation is used for training 271 and testing the GA-BPNN model. The hidden layer function used in this paper is the hyperbolic 
272 tangent sigmoid transfer function (tansig), and the output layer function is the Log-sigmoid transfer 273 function (logsig).

274 The parameters setting of the K-fold cross-validation is: $\mathrm{K}=3, \mathrm{~N}=150$. That is, 150 samples of 275 experimental data are randomly divided into 3 groups, and 2 groups are selected as the training 276 data of the GA-BPNN in turn, and the remaining 1 group is used as the testing data. So, the 277 recognition rate of each test is recorded and the final result is the average of 3 recognition rates.

\section{Results \& Discussion}

\section{Experimental data analysis}

280 Four typical waveform samples of raw detection signals are randomly selected from the 281 experimental data, and their last period data are drawn in Fig. 9

282 The figure shows the similarities and differences of the ultrasonic propagating in the concrete test 283 block. Based on the physical mechanism of the ultrasonic propagation, the different diameters of 284 holes are the main reason for the difference between ultrasonic detection signal waveforms. In 285 addition, the sizes and the shapes of gravel at different locations are different in the concrete, which 286 is another important reason for the different detection waveforms (Garnier et al., 2009).

287 Based on the reconstructed data, five features extracted from 150 signals are calculated. The five 288 features are separately shown in Figs. 10-14.

289 Five features of the reconstructed defective and defect-free signals do not show obvious regularity 290 or organization from Figs. 10-14. The figures show that the feature values are different more or 291 less even they are extracted from the same defect shared the same diameters of penetrating holes, 292 or at the same detection points. Five features are aliasing and these reconstructed signals are 293 inseparable linearly based on the mere measurement of single feature. On the one hand, the uneven 294 distribution of coarse aggregate in concrete will generate acoustic measurement uncertainty, and 295 that causes the complexity of ultrasonic detection signal. In particular, it is a non-linear, non296 stationary signal and contains many mutational components. On the other hand, the stability and 297 accuracy of the hardware system influence the output deviation, so the detection signals exist a 298 certain distortion inevitably. Nevertheless, it can be seen that partial feature data are distributed 299 centrally, such as the kurtosis coefficient of $9 \mathrm{~mm}$ defect detection data in Fig. 12. Although 300 Different detection signals have similarities on a single feature, we can distinguish differences 301 between different signals on multiple features fusion. Then, five features are regarded as essential 302 characteristics for the classification of defects in this paper.

303 Comparison

304 The optimal solution is used to initialize the configuration parameters for the proposed GA-BPNN 305 algorithm. The optimal number of hidden layer nodes of BPNN calculated by GA with the three306 fold cross-validation method is 12 , and then the number of each layer's nodes is 5,12 , and 2.

307 To demonstrate the advantages and disadvantages of the GA-BPNN, a BPNN without optimization 308 is utilized for algorithmic performance analysis, and we further draw their convergent curves. We 309 use a default function in the neural network toolbox to initialize weights and biases, with 11 hidden 310 layer nodes, according to the empirical rule $(2 * 5+1)$ in the paper of Guo Z.H. et al. (Guo et al., 311 2011). Some parameters of the BPNN include the number of input nodes, the number of output 
312 nodes, the training stop condition, learning rate and activating function are the same as the 313 parameters setting of GA-BPNN in Section 3.

314 The training error curves and test error curves of the computational processes are painted in Figs.

315 15-16, where two figures show how the outputs of models converge to the actual tag value. The 316 feature data picked up for operating and drawing the curves are randomly selected from the training 317 dataset and the test dataset respectively. The error set by the BPNN in this paper is 0.001 , and the 318 epochs required by BPNN are more than twice that of GA-BPNN. The computational cost of the 319 BPNN is higher than that of GA-BPNN. In addition, the GA-BPNN also converges faster in the 320 early stage of operation. In Figs. 15-16, it can be seen from the mean squared error curves that the 321 GA-BPNN takes fewer epochs under the same termination conditions. The GA-BPNN has higher 322 operating efficiency and convergence speed to approach the model's predictive values. The 323 statistical results on 100 training data calculated by GA-BPNN with the three-fold cross-validation 324 are shown in Table 1, the statistical results on the 50 test data are shown in Table 2.

325 Although the convergence speed of GA-BPNN is higher, it has to spend much time to solve the 326 optimum in the training stage, i.e., it is about 489.049 seconds to search the optimum. Its average 327 training time is about 0.0993 seconds and the average test time is about 0.0053 seconds. 328 Correspondingly, the average training time of BPNN is about 0.1413 seconds and its average test 329 time is about 0.0057 seconds. Its test recognition accuracy is about $86.67 \%$ which is less than the 330 recognition accuracy of GA-BPNN.

331 Three defect recognition accuracies from the three-fold cross-validation are all higher than $90 \%$ 332 shown in the statistical results, which can prove the extracted features are effective in 333 characterizing the presence or absence of defects in concrete, and the GA-BPNN is feasible as a 334 concrete defect-recognition model. Furthermore, the proposed method can identify the defects 335 automatically from detection data, then operators do not need to possess professional detection 336 knowledge for reading and identifying recognition results. It is quite important for its practical 337 engineering applications.

338 According to previous research on the recognition method of concrete ultrasonic detection signal, 339 we choose radial basis function network (RBF) (Chen \& Ma, 2011) and support vector machine 340 (SVM) (Saechai, Kongprawechnon \& Sahamitmongkol, 2012) using the data in this paper to carry 341 out classification experiments. Also, under the 3-fold cross-validation, 150 concrete ultrasonic data 342 consisting of 5 features are used. The results of the comparative experiment are shown in Table 3. 343 The recognition accuracy of SVM, RBF, and BPNN methods have little differences, but none of 344 them reaches $90 \%$. Compared with previous studies, the size of the concrete defects in this paper 345 are smaller and therefore the detection signal is more challenging to be identified. The method we 346 proposed is more accurate than the above three methods.

347 It is shown that the proposed method leads to the performance approaching high recognition 348 accuracy. When measuring the acoustic, the degree of adhesion and contact force of the ultrasonic 349 probe to the concrete surface may cause the recognition error due to the fact that concrete is a 350 complex and multi-phase medium. Therefore, the obtained detection signals are complex and 
351

352

353

354

355

356

357

358

359

360

361

362

363

364

365

366

367

368

369

370

371

372

373

374

375

376

377

378

379

380

381

382

383

384

385

386

387

388

389

diverse. Although it is hard to completely identify all modes of the complex ultrasonic detection signals from concrete, more defect-type will be further investigated as our future works.

\section{Conclusions and future work}

In order to recognize the concrete defects with high reliability and accuracy by using ultrasonic testing signals, we propose an intelligent method which includes a signal processing sub-algorithm and a recognition sub-algorithm. We extract fundamental information from the first node of the third layer by using wavelet packet transform (WPT) and calculate five feature variables of the reconstructed signals. Moreover, the GA-BPNN-based sub-algorithm identifies the concrete defects, where GA optimized BP neural network (GA-BPNN) model has been proposed embedding a K-fold cross-validation method. As a practical application of a typical type of hole defects in concrete, we utilize the method to identify the defects in a C30 class concrete test block. Based upon the test points, we obtained 150 ultrasonic detection signals containing no defect and hole defects at various locations, and then performed identification experiments based on these data sets using the method in this paper. GA-BPNN has higher diagnosis accuracy and faster running speed than existing methods. The experimental results show the effectiveness of the proposed method while the concrete hole defects have been recognized with high accuracy.

In the future, we will further verify the effectiveness of this method in more types of concrete defect (e.g., cracks and foreign matter) ultrasonic detection signal data and develop more effective methods to solve complex problems in the field (Mittal et al., 2020.), such as characteristic indexes, optimizer, machine learning. Then these effective methods will be extended to more detection signal fields. Simultaneously, the sensor network solution is also our future directly for information fusion (Naeem et al.,2021). Simultaneously, the uneven distribution of coarse aggregate could be considered as a stochastic distribution optimization problem (Ren, Zhang \& Zhang, 2019), its influence on the accuracy of detection signal recognition is another theoretical perspective for our future works.

\section{Acknowledgements}

We thank two units for their help in designing the hardware system and the actual parameters testing of the ultrasonic probe, Hangzhou Ruidian Meter Co., Ltd. and Shanghai Ultra Precision Motion Control and Detection Engineering Research Center.

\section{References}

Bien J, Kaminski T, Kuzawa M. 2019. Taxonomy of non-destructive field tests of bridge materials and structures. Archives of Civil and Mechanical Engineering 19:1353-1367.

Schabowicz K. 2019. Non-destructive testing of materials in civil engineering. Material 12:3237.

Janku M, Cikrle P, Grosek J, Anton O, Stryk J. 2019. Comparison of infrared thermography, ground-penetrating radar and ultrasonic pulse echo for detecting delaminations in concrete bridges. Construction and Building Materials 225:1098-1111.

Ushakov VM, Davydov DM. 2013. A review of international (ISO) and European (EN) standards for the ultrasonic testing of welded joints. Russian Journal of Nondestructive Testing 49:6166 . 
390

391

392

393

394

395

396

397

398

399

400

401

402

403

404

405

406

407

408

409

410

411

412

413

414

415

416

417

418

419

420

421

422

423

424

425

426

427

428

429

Ozsoy U, Koyunlu G, Ugweje OC. 2017. Nondestructive Testing of Concrete using Ultrasonic Wave Propagation. In Proceedings of 13th International Conference on Electronics, Computer and Computation (ICECCO), Abuja, Nigeria, 28-29.

Tibaduiza-Burgos DA, Torres-Arredondo MA. 2015. Investigation of an expert health monitoring system for aeronautical structures based on pattern recognition and acoustoultrasonics. Smart Materials and Structures 24:1-18.

Cheema A, Singh M. 2019. Psychological stress detection using phonocardiography signal: An empirical mode decomposition approach. Biomedical Signal Processing and Control 49:493505.

Combet F, Gelman L, LaPayne G. 2012. Novel detection of local tooth damage in gears by the wavelet bicoherence. Mechanical Systems and Signal Processing 26:218-228.

Zhu Q, Wang YS, Shen GQ. 2011. Comparison and application of time-frequency analysis methods for nonstationary signal processing. Advanced Research on Computer Education, Simulation and Modeling, PTI 175:286-291.

Liu SY, Li ZP, Wu T, Zhang W. 2021. Determining ultrasound arrival time by HHT and kurtosis in wind speed measurement. Electronics 10:523.

Kumar A, Kumar R. 2019. Role of Signal Processing, Modeling and decision making in the diagnosis of rolling element bearing defect: a review. Journal of Nondestructive Evaluation 38:5.

Bochud N, Gomez AM, Rus G, Peinado AM. 2015. A sparse digital signal model for ultrasonic nondestructive evaluation of layered materials. Ultrasonics 62:169-173.

Iyer S, Sinha SK, Tittmann BR, Pedrick MK. 2012. Ultrasonic signal processing methods for detection of defects in concrete pipes. Automation in Construction 22:135-148.

Naeem A, Abdul RJ, Muhammad R, Sidra A, Jerry CWL, \& Thippa RG. 2021. DARE-SEP: A hybrid approach of distance aware residual energy-efficient SEP for WSN. IEEE Transactions on Green Communications and Networking 5(2):611-621.

Rajput DS, Basha SM, Xin Q, Gadekallu TR, Kaluri R, Lakshmanna K, Maddikunta PKR. 2021. Providing diagnosis on diabetes using cloud computing environment to the people living in rural areas of India. Journal of Ambient Intelligence Humanized Computing doi: 10.1007/s12652-021-03154-4.

Xu YD, Jin RY. 2018. Measurement of reinforcement corrosion in concrete adopting ultrasonic tests and artificial neural network. Construction and Building Materials 177:125-133.

Acciani G, Brunetti G, Fornarelli G, Giaquinto A. 2010. Angular and axial evaluation of superficial defects on non-accessible pipes by wavelet transform and neural network-based classification. Ultrasonics 50:13-25.

Rodriguesa LFM, Cruzb FC, Oliveirac MA, Simas EF, Albuquerqued MCS, Silvad IC, Fariasd CTT. 2019. Carburization level identification in industrial HP pipes using ultrasonic evaluation and machine learning. Ultrasonics 94:145-151.

Saechai S, Kongprawechnon W, Sahamitmongkol R. 2012. Test system for defect detection in construction materials with ultrasonic waves by support vector machine and neural network. 
430

431

432

433

434

435

436

437

438

439

440

441

442

443

444

445

446

447

448

449

450

451

452

453

454

455

456

457

458

459

460

461

462

463

464

465

466

467

In Proceedings of 6th International Conference on Soft Computing and Intelligent Systems (SCIS), Kobe, Japan, 20-22, November, pp.1034-1039.

Chen Y, Ma HW. 2011. Application of artificial neural network to flaw classification in ultrasonic testing. Advanced Materials Research 328:1876-1880.

Zhang KX, Lv GL, Guo SF, Chen D, Liu YJ, Feng W. 2020. Evaluation of subsurface defects in metallic structures using laser ultrasonic technique and genetic algorithm-back propaga-tion neural network. NDT \& E International 116:102339.

Shrestha A, Mahmood A. 2019. Review of deep learning algorithms and architectures. IEEE Access 7:53040-53065.

Wang XP. 2015. Research and optimization of BP neural network algorithm. In Proceedings of 2015 Seventh International Conference on Measuring Technology and Mechatronics Automation (ICMTMA 2015), Nanchang, China, 13-14 June, pp.818-822.

Slonski M, Schabowicz K, Krawczyk E. 2020. Detection of flaws in concrete using ultrasonic tomography and convolutional neural networks. Materials 13:1557.

Babouri MK, Ouelaa N, Kebabsa T, Djebala A. 2019. Application of the cyclostationarity analysis in the detection of mechanical defects: comparative study. International Journal of Advanced Manufacturing Technology 103:1681-1699.

Kim HJ, Mission JL, Dinoy PR, Kim HS, Park TW. 2020. Guidelines for impact echo test signal interpretation based on wavelet packet transform for the detection of pile defects. Applied Sciences 10:2633.

Schimmack M, Mercorelli P. 2018. A wavelet packet tree denoising algorithm for images of atomic-force microscopy. Asian Journal of Control 20:1-12.

Samaratunga D, Jha R, Gopalakrishnan S. 2016. Wavelet spectral finite element for modeling guided wave propagation and damage detection in stiffened composite panels. Structural Health Monitoring-an International Journal 15:317-334.

Cruz FC, Simas Filho EF, Albuquerque MCS, Silva IC, Farias CTT, Gouvêa LL. 2016. Efficient feature selection for neural network based detection of flaws in steel welded joints using ultrasound testing, Ultrasonics 73:1-8.

Wan P, Li QF. 2014. Wavelet transform-based feature extraction for ultrasonic flaw signal classification. Neural Computing \& Applications 24:817-826.

Wang XK, Guan SY, Hua L, Wang B, He XM. 2019. Classification of spot-welded joint strength using ultrasonic signal time-frequency features and PSO-SVM method. Ultrasonics 91:161169.

Virmani J, Kumar V, Kalra N, Khandelwal N. 2013. SVM-based characterization of liver ultrasound images using wavelet packet texture descriptors. Journal of Digital Imaging 26:530543.

Bagan H, Takeuchi W, Yamagata Y, Wang XH, Yasuoka Y. 2009. Extended averaged learning subspace method for hyperspectral data classification. Sensors 9:4247-4270.

Peer] Comput. Sci. reviewing PDF | (CS-2021:04:60010:1:1:NEW 9 Jun 2021) 
468

469

470

471

472

473

474

475

476

477

478

479

480

481

482

483

484

485

486

487

488

489

490

Peng JW, Lv WH, Xing HY, Wu XJ. 2013. Temperature compensation for humidity sensor based on improved GA-BP neural network. Chinese Journal of Scientific Instrument 34:153160.

Han ZY, Huang XG. 2019. GA-BP in thermal fatigue failure prediction of microelectronic chips. Electronics 8:542.

Guo ZH, Wu J, Lu HY, Wang JZ. 2011. A case study on a hybrid wind speed forecasting method using BP neural network. Knowledge-Based Systems 24:1048-1056.

Garnier V, Chaix JF, Rossat M, Payan C, Corneloup G. 2009. Non destructive characterisation of concretes by ultrasonic wave's propagation. Mecanique \& Industries 10:299-303.

Ren M, Zhang Q, Zhang J. 2019. An introductory survey of probability density function control. Systems Science \& Control Engineering 7:158-170.

Shi YB, Zhang JJ, Jiao JJ, Zhao R, Cao HL. 2021. Calibration analysis of high-G MEMS accelerometer sensor based on wavelet and wavelet packet denoising. Sensors 21:1231.

Zhang G, Duffy AP, Orlandi A. 2017. Statistical figures of merit for the feature selective validation method. IEEE Transactions on Electromagnetic Compatibility 59:1482-1489.

Javed AR, Usman M, Rehman SU, Khan MU, Haghighi MS. 2020. Anomaly detection in automated vehicles using multistage attention-based convolutional neural network. IEEE Transactions on Intelligent Transportation Systems doi: 10.1109/TITS.2020.3025875.

Mittal M, Iwendi C, Khan S, Javed AR. 2020. Analysis of security and energy efficiency for shortest route discovery in low-energy adaptive clustering hierarchy protocol using LevenbergMarquardt neural network and gated recurrent unit for intrusion detection system. Transactions on Emerging Telecommunications Technologies doi: 10.1002/ett.3997. 
Figure 1

Three-layer wavelet packet decomposition diagram.

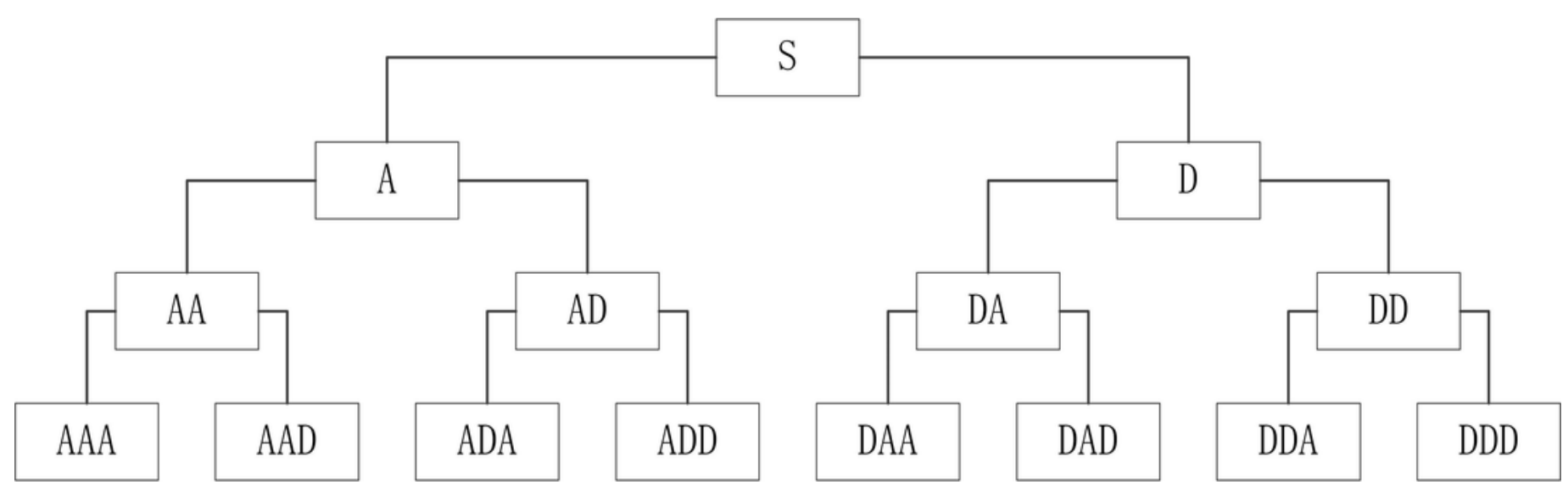


Figure 2

BP neural network structure

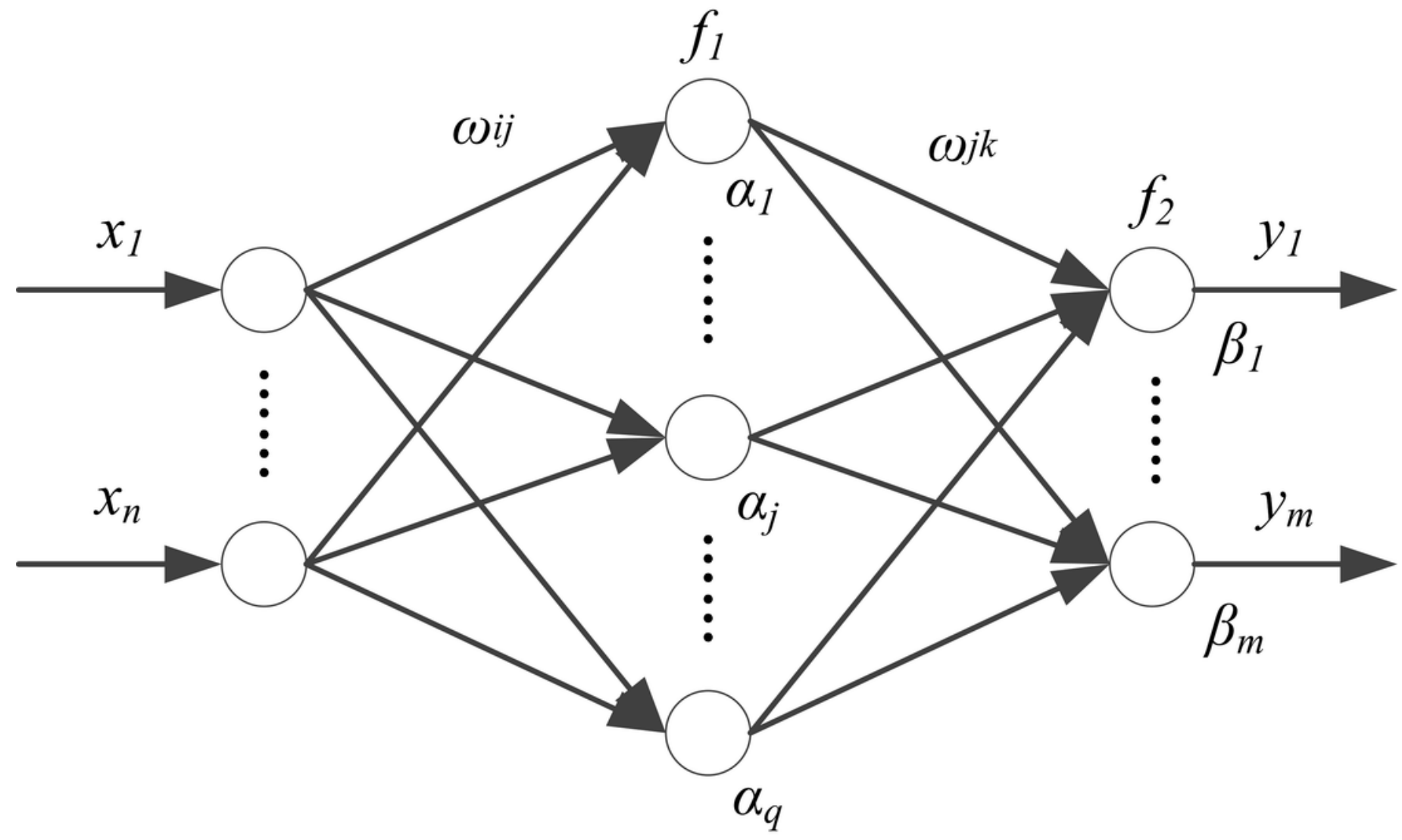


Figure 3

Parameters coding sequence

\begin{tabular}{|c|c|c|c|c|}
\hline hidden layer nodes & $\omega_{\mathrm{ij}}$ & $\alpha_{j}$ & $\omega_{\mathrm{jk}}$ & $\beta_{\mathrm{m}}$ \\
\hline $\mathrm{q}$ & $n \cdot\left(2^{q}-1\right)$ & $2^{\mathrm{q}}-1$ & $\left(2^{\mathrm{q}}-1\right) \cdot \mathrm{m}$ & $\mathrm{m}$ \\
\hline
\end{tabular}


Figure 4

algorithm flowchat 


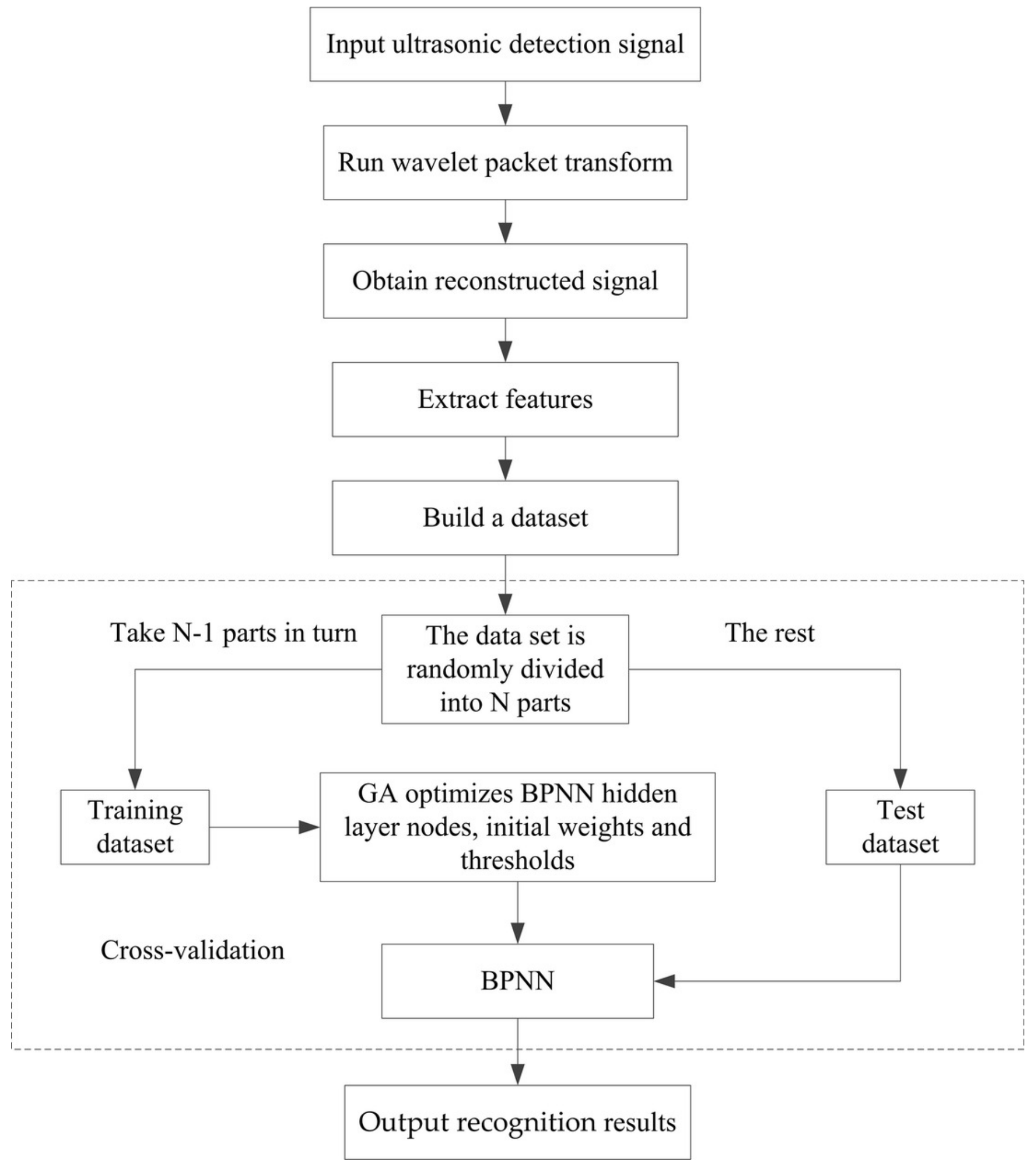


Figure 5

The schematic of the experimental setup for evaluating concrete defects with ultrasonic

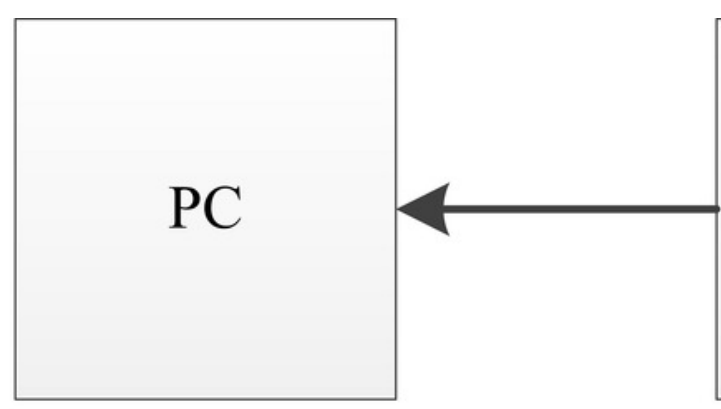

Analog-todigital conversion

\section{conversion}
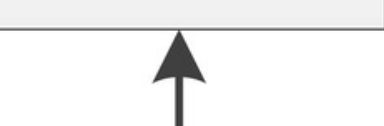

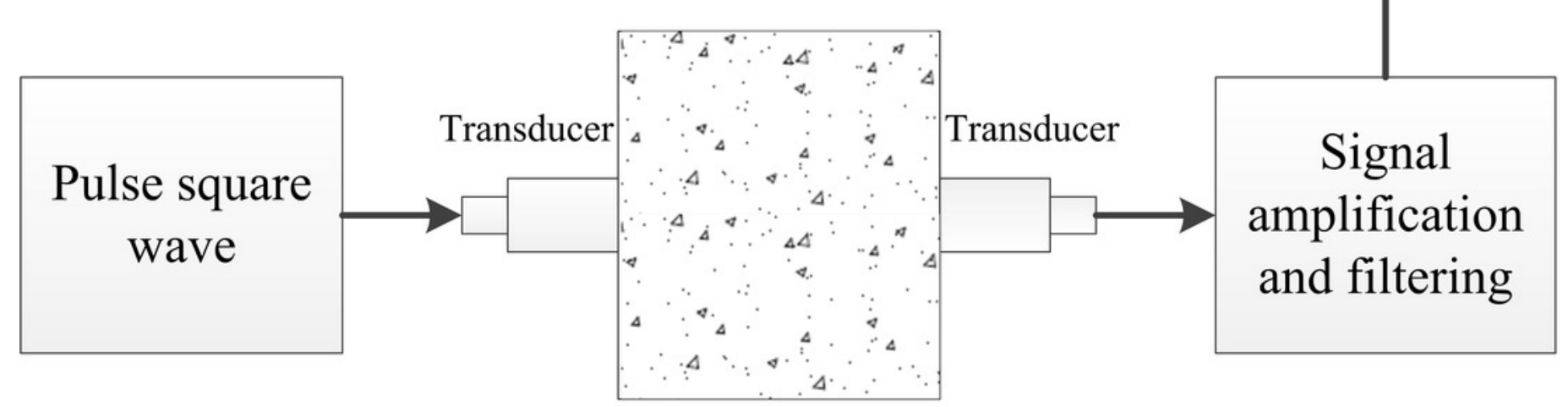

Concrete 


\section{Figure 6}

Concrete test block

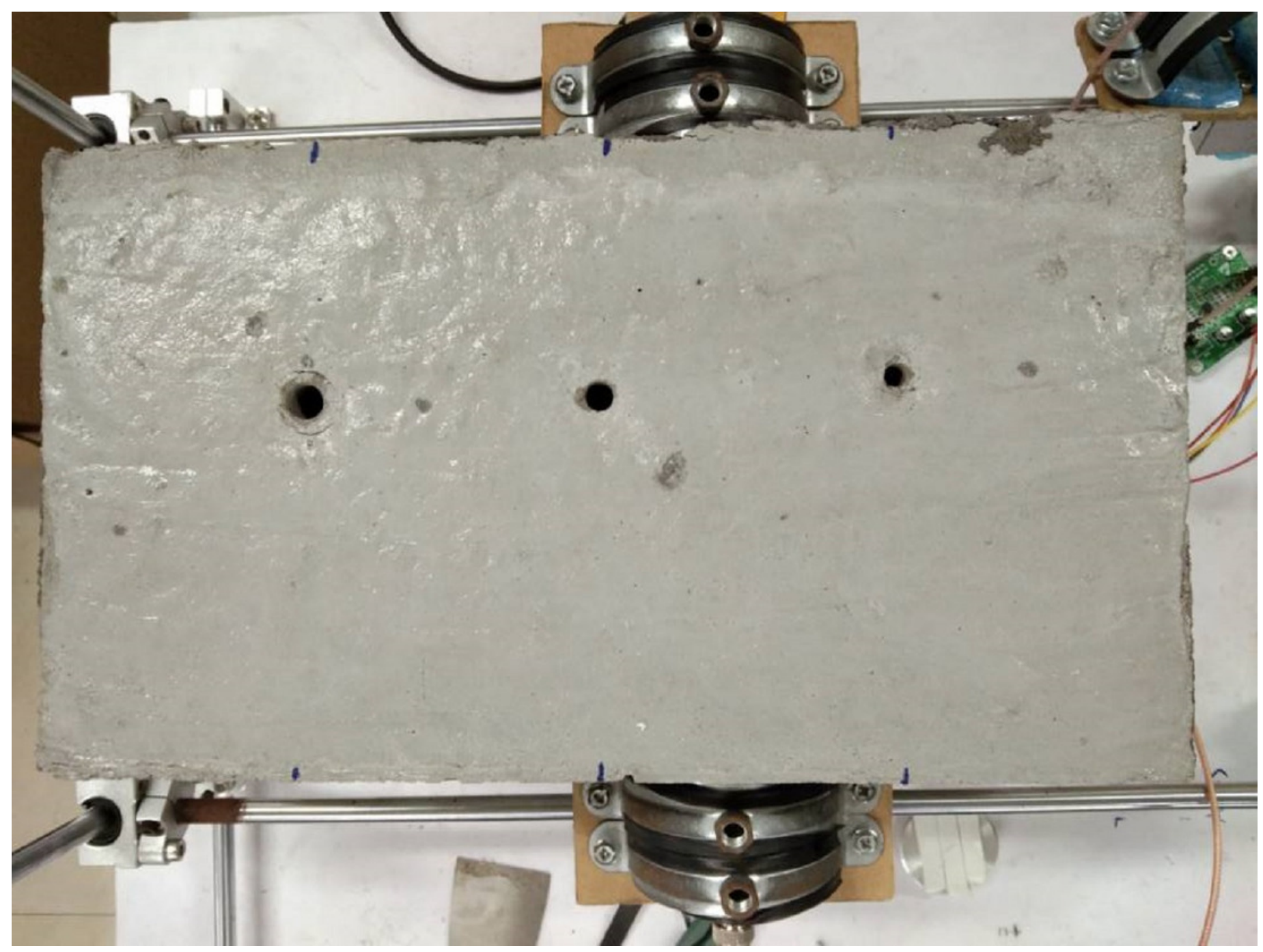




\section{Figure 7}

detection location diagram

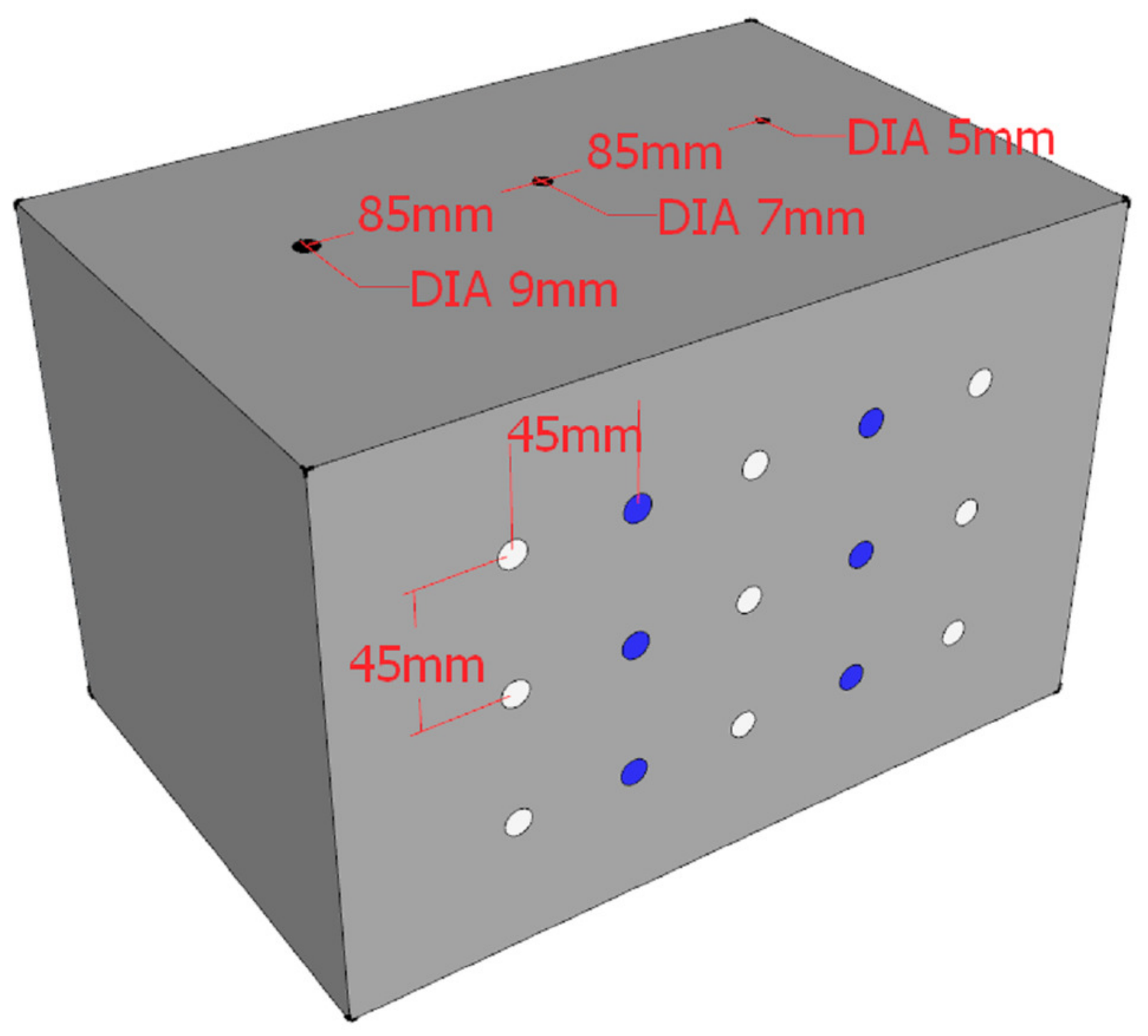




\section{Figure 8}

Diagram of the used experimental setup

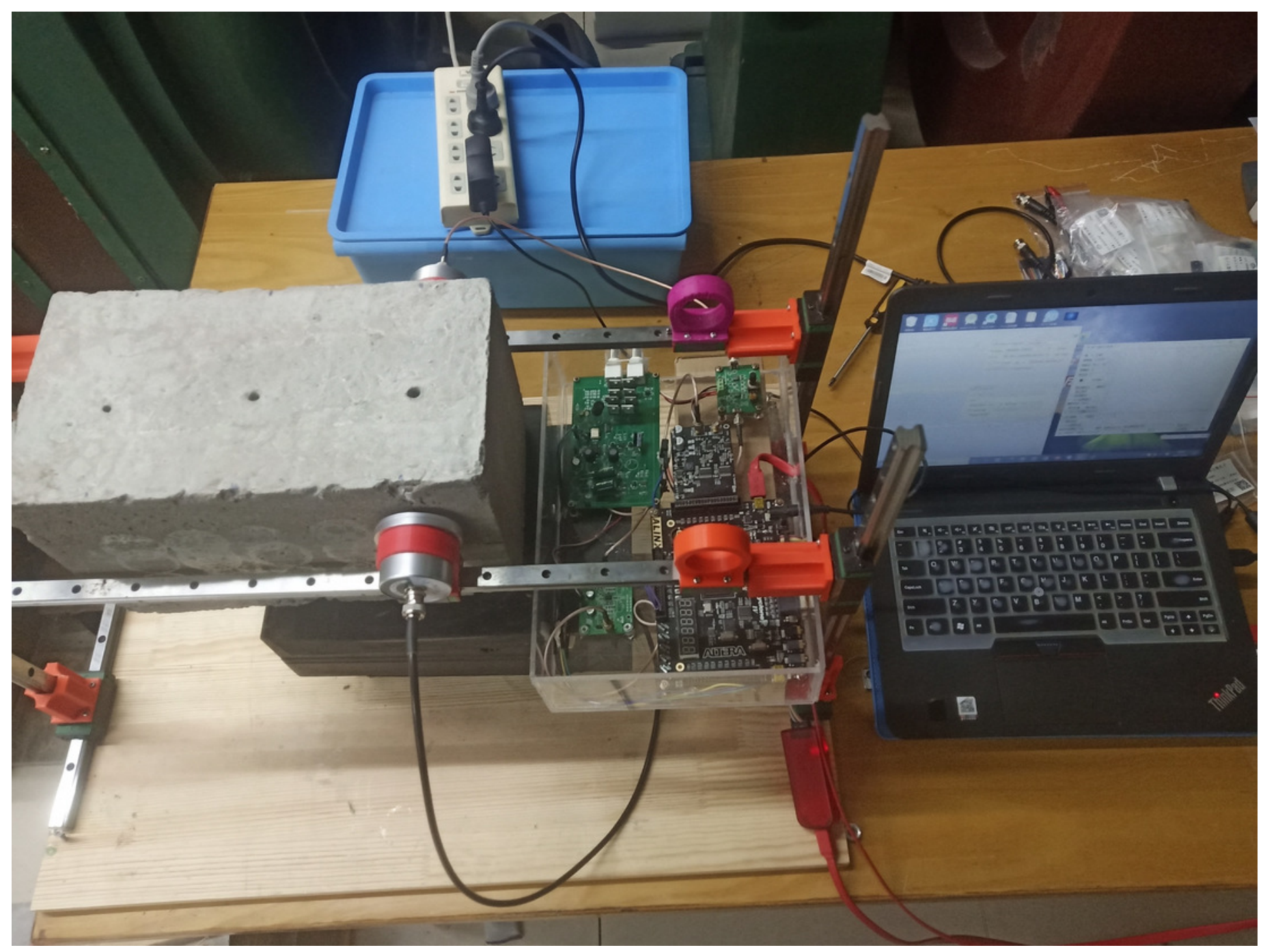




\section{Figure 9}

Four types of ultrasonic detection signal waveforms

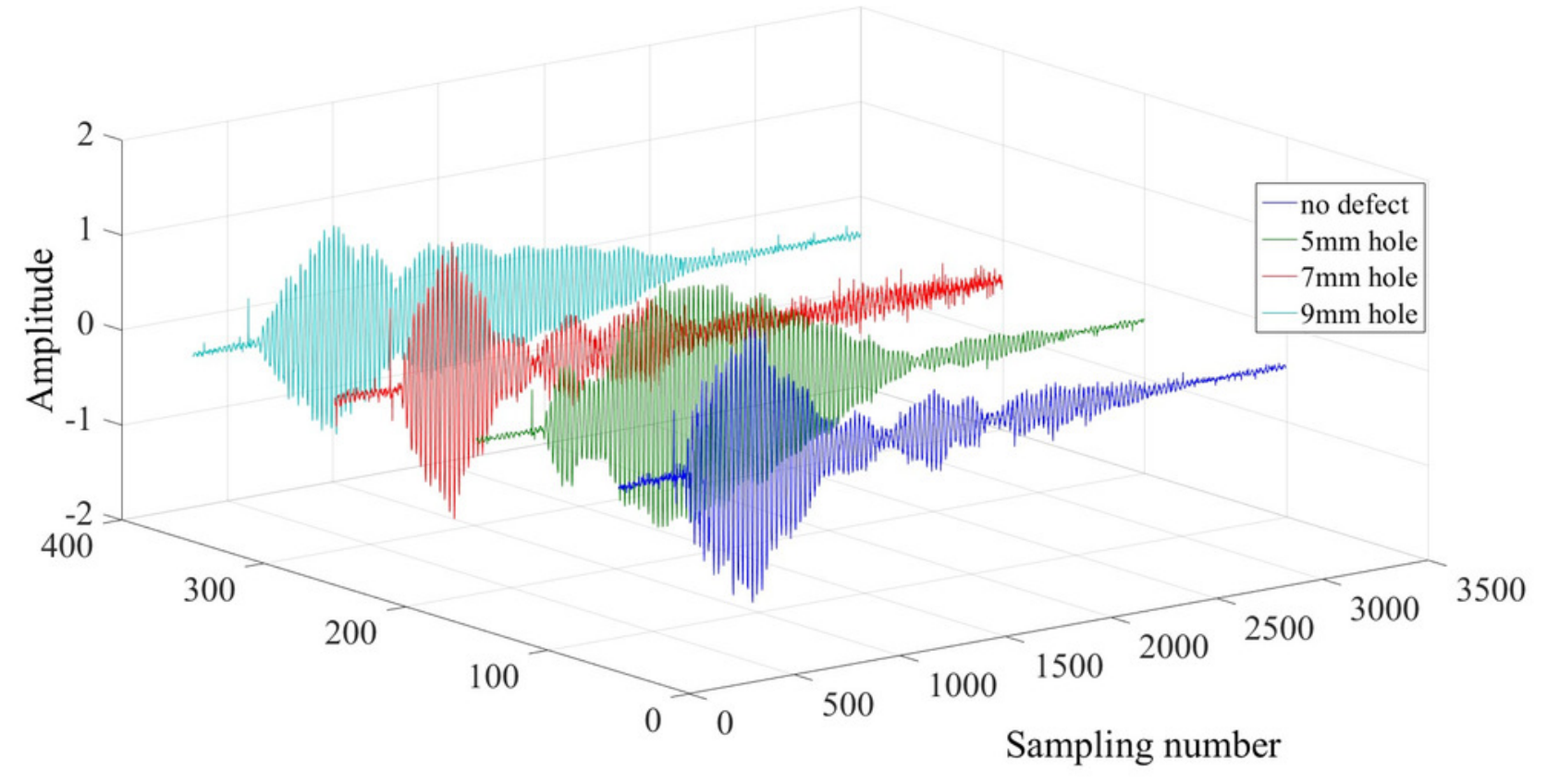


Figure 10

Mean distribution

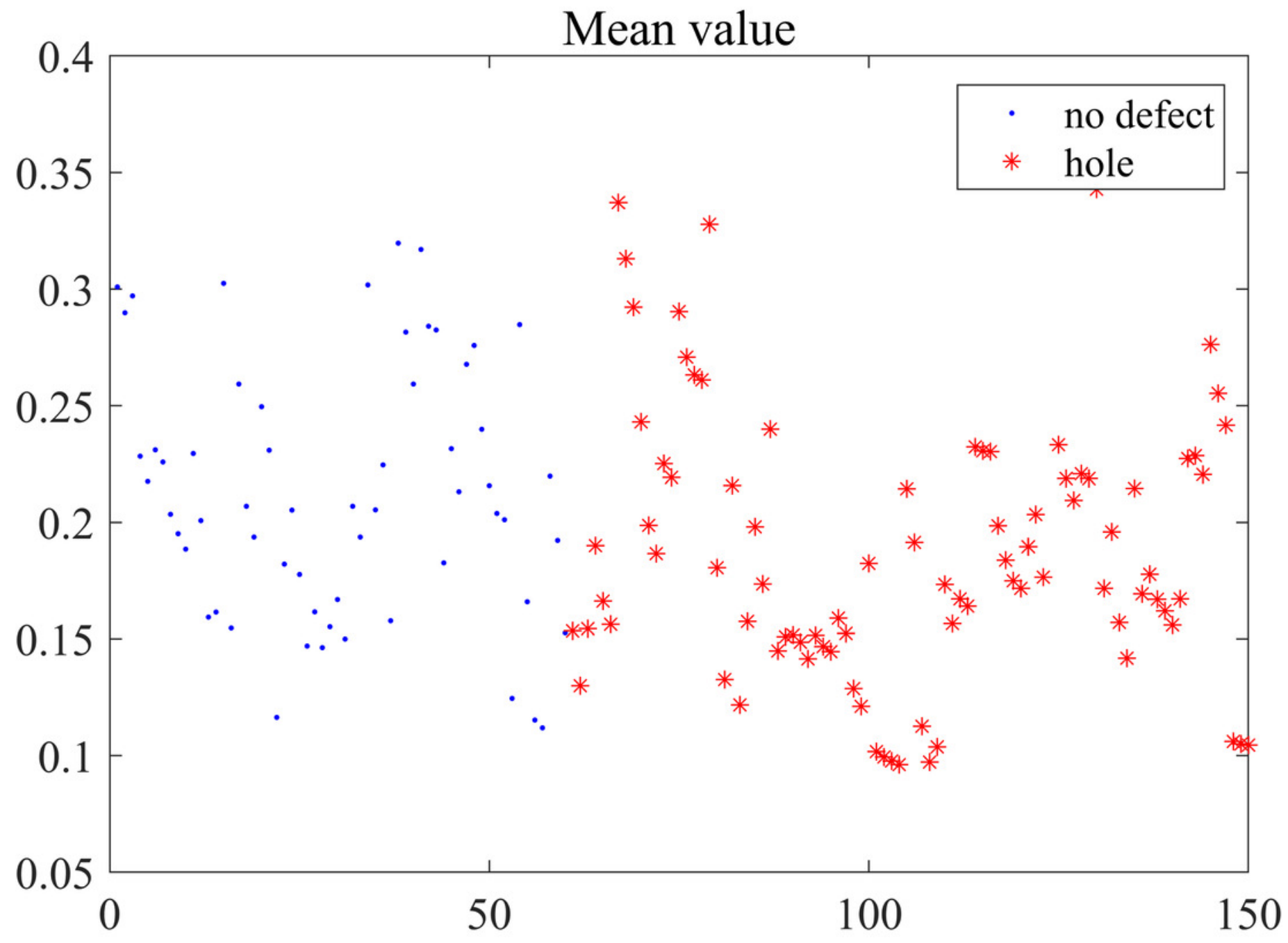


Figure 11

standard deviation distribution

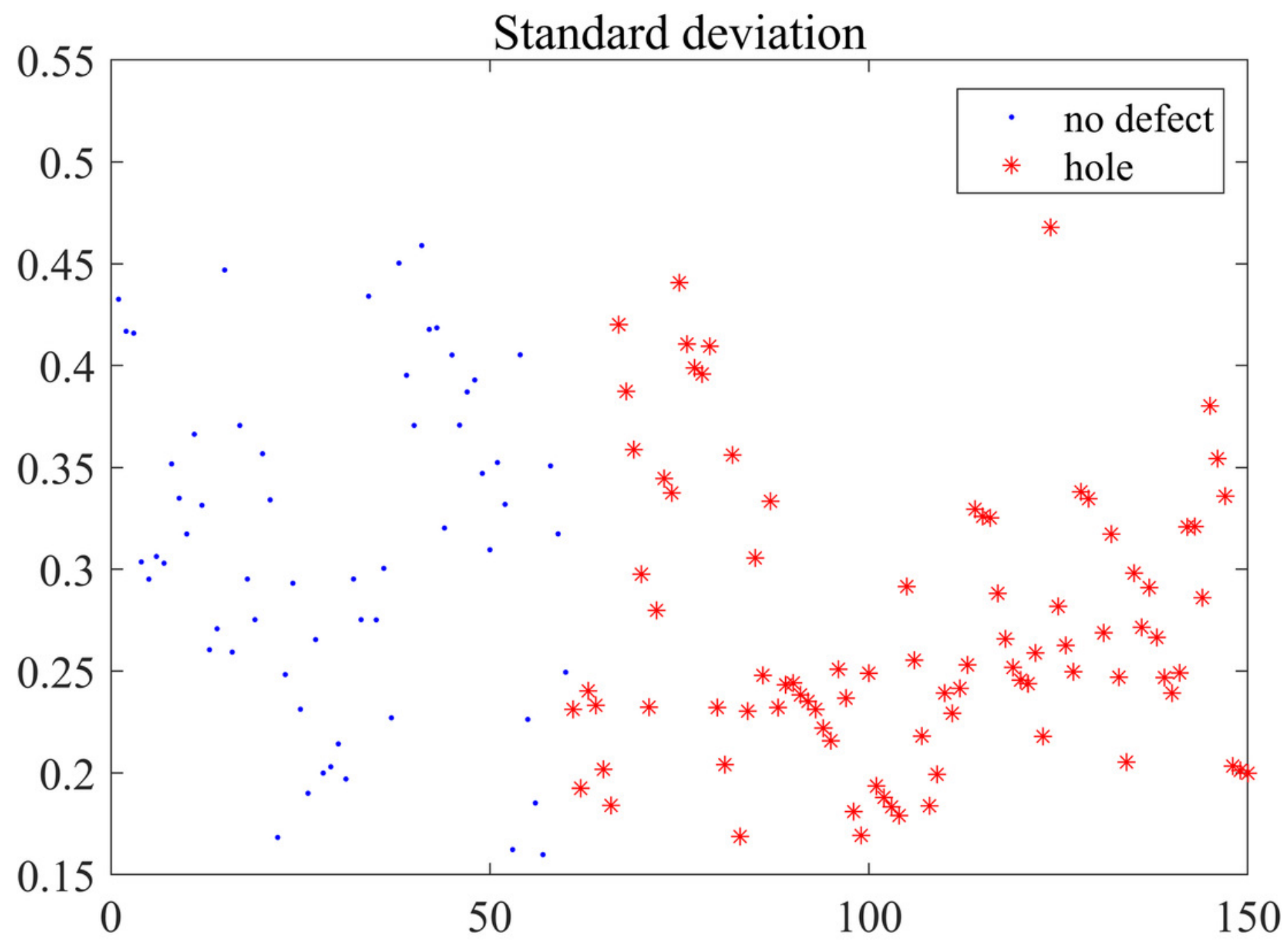


Figure 12

kurtosis coefficient distribution

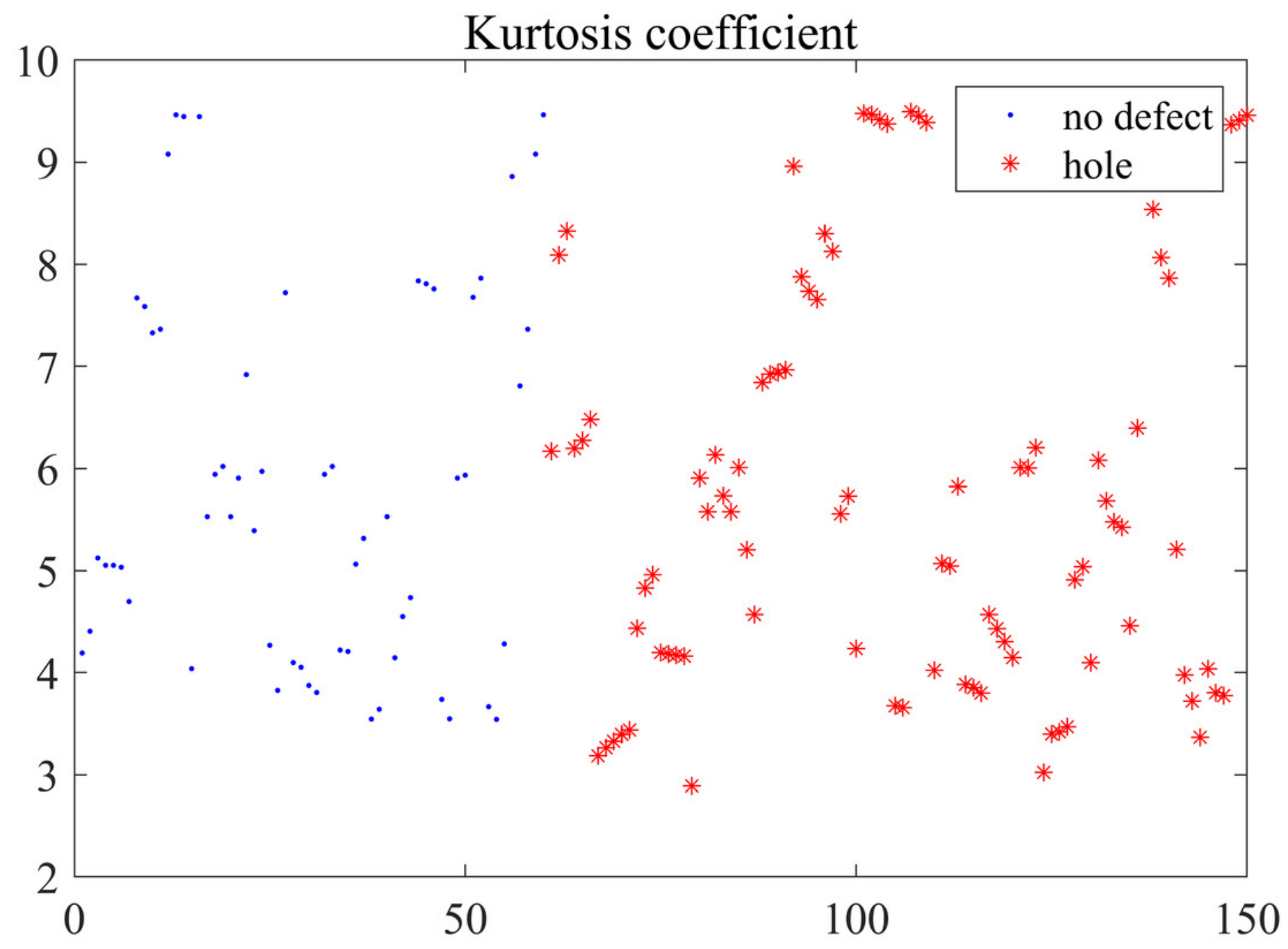


Figure 13

skewness coefficient distribution

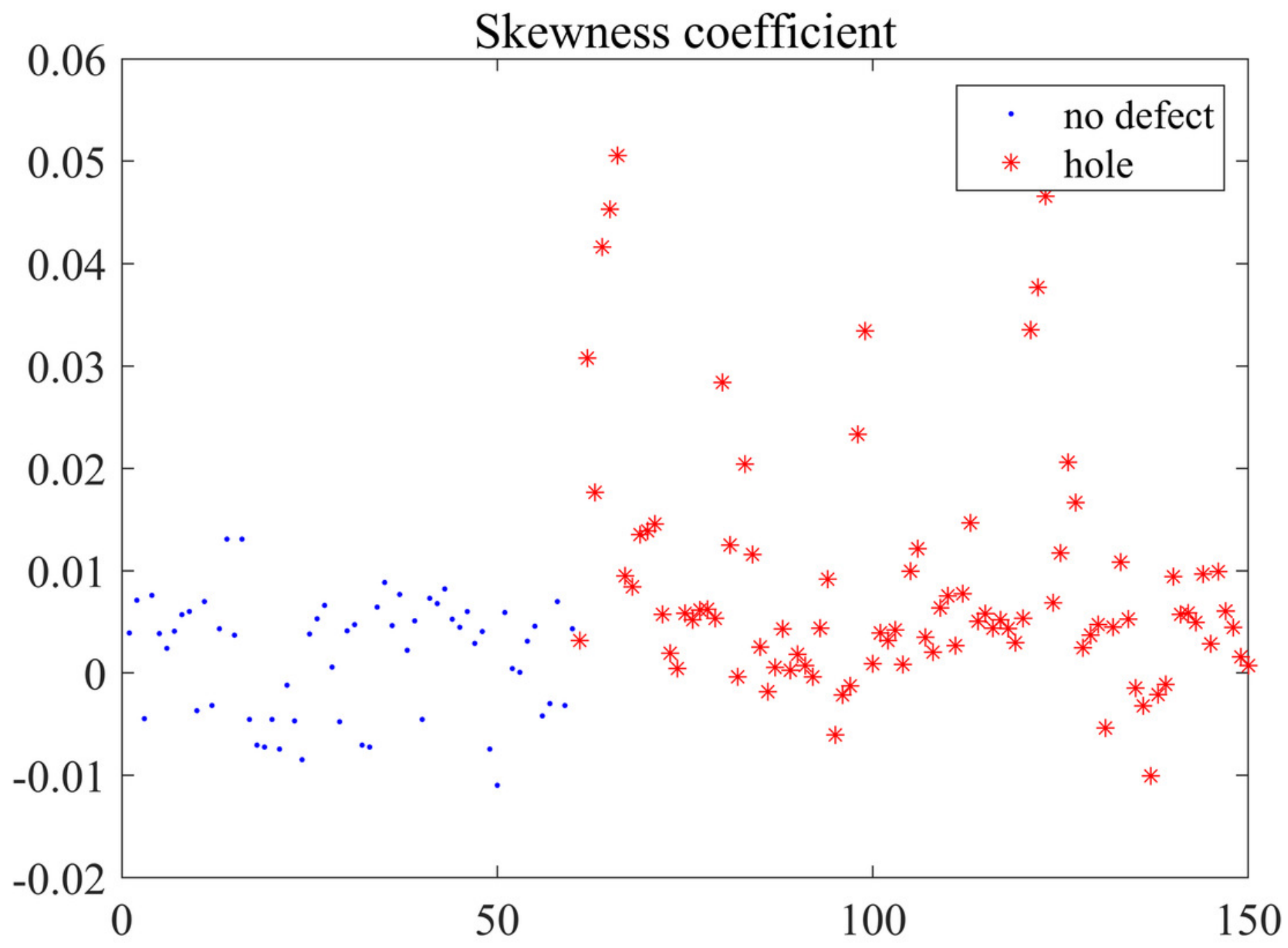


Figure 14

energy ratio distribution

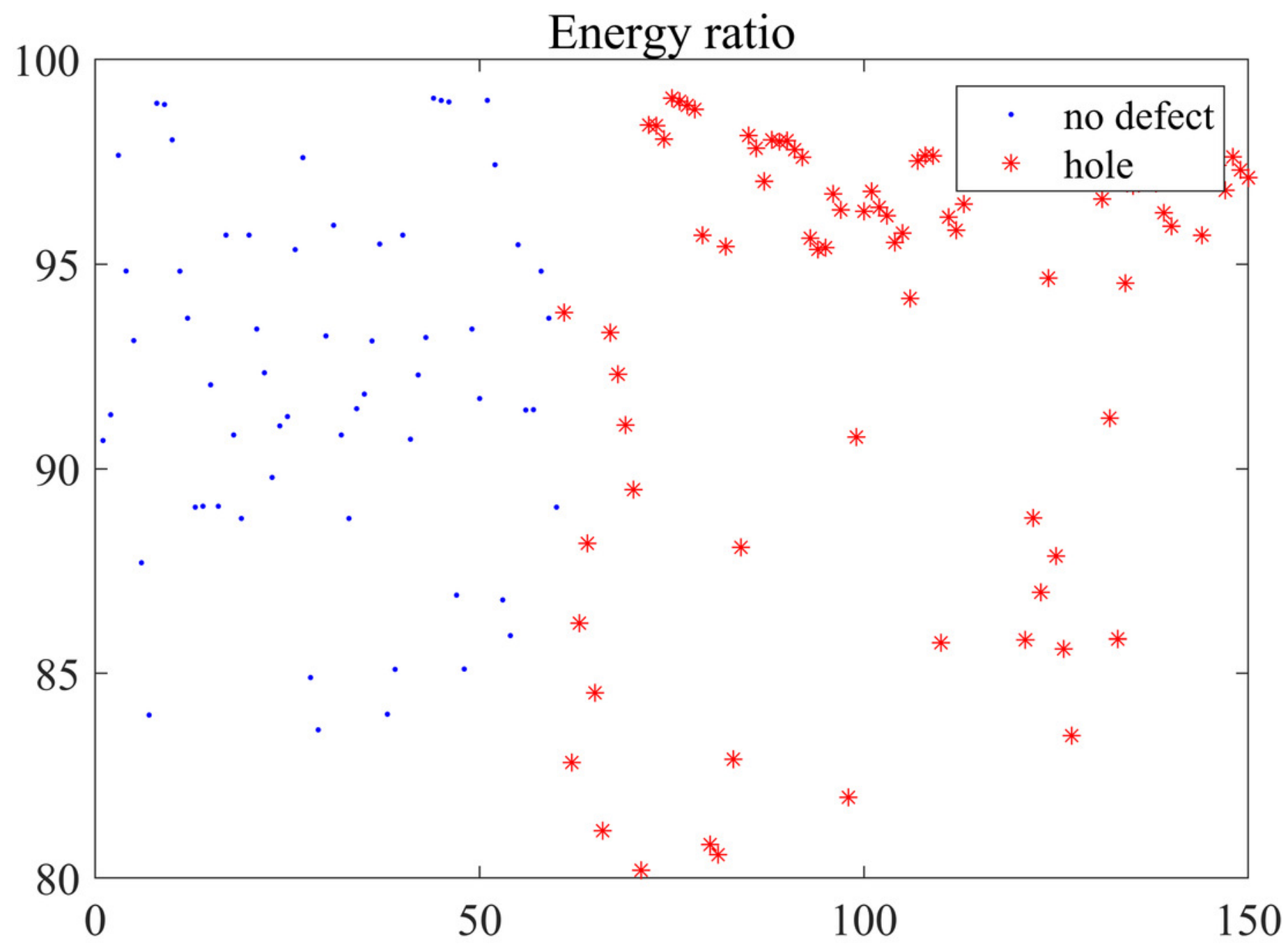


Figure 15

The error curves in training dataset

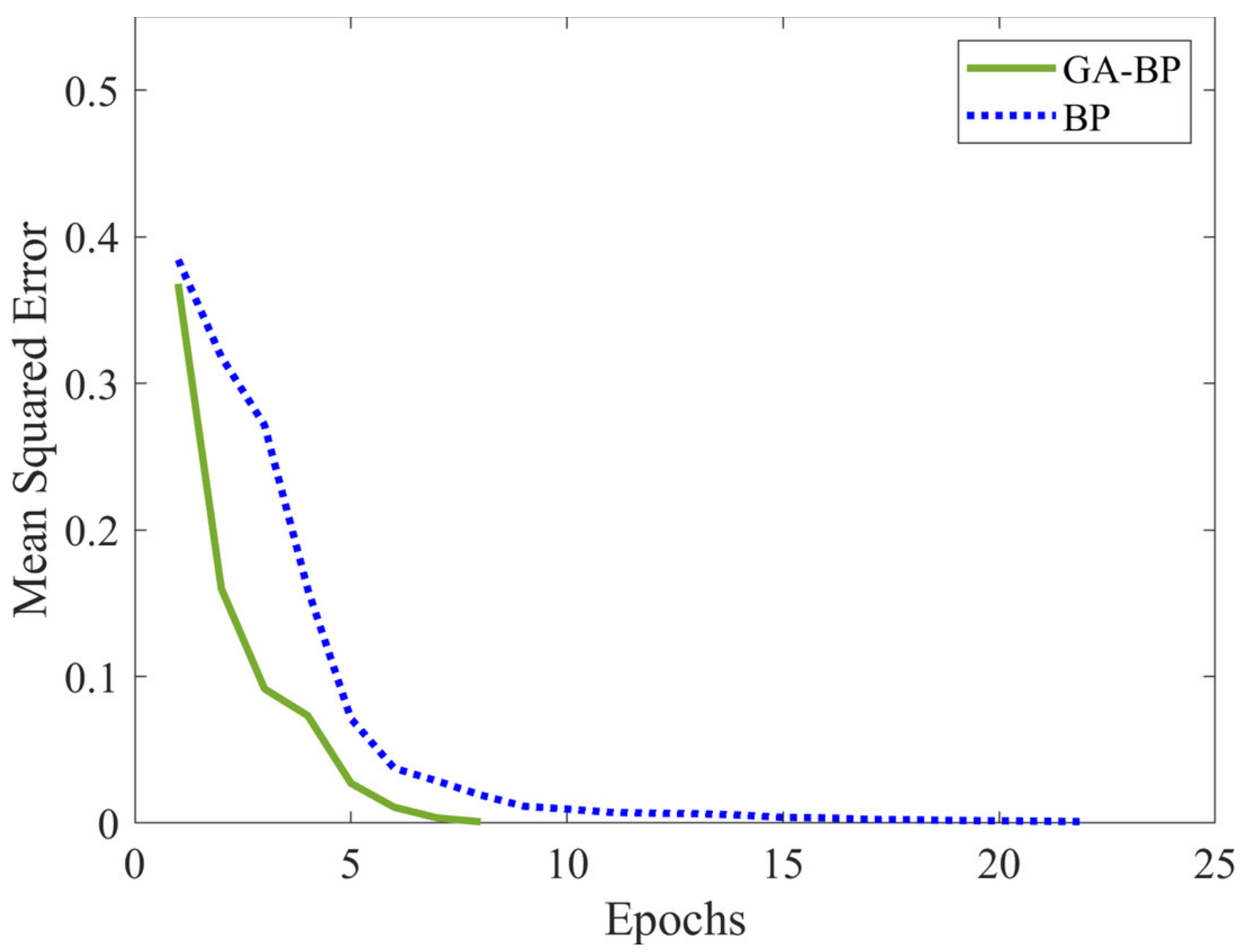


Figure 16

The error curves in test dataset

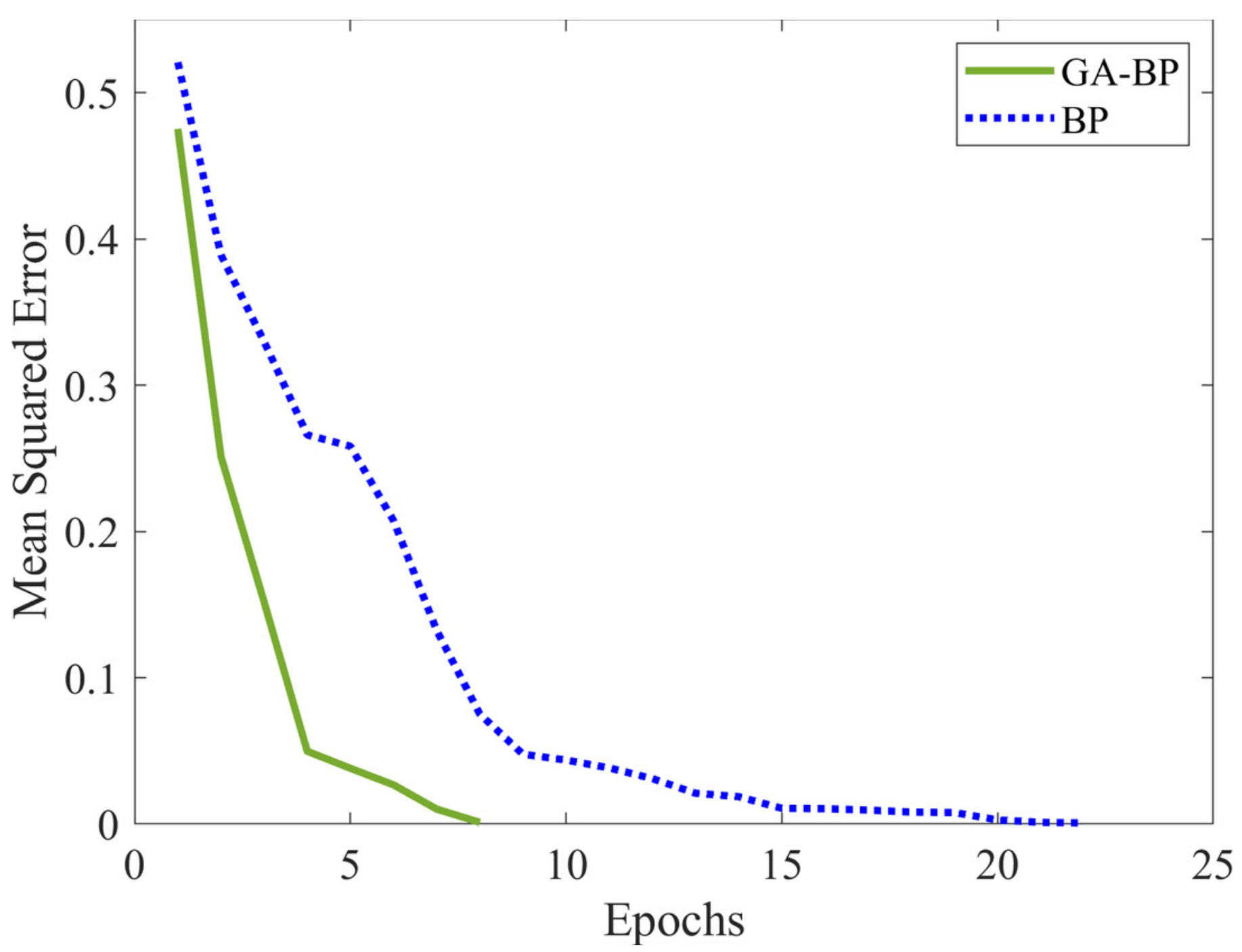




\section{Table $\mathbf{1}$ (on next page)}

Training dataset recognition results 


\begin{tabular}{|c|c|c|c|c|c|c|}
\hline \multirow{2}{*}{ No. } & \multirow{2}{*}{ Class } & & \multicolumn{2}{|c|}{ Recognition result } & \multirow{2}{*}{$\operatorname{Accuracy}(\%)$} & \multirow{2}{*}{ Average Accuracy(\%) } \\
\hline & & & True & False & & \\
\hline \multirow{4}{*}{1} & No defect & & 38 & 2 & \multirow{4}{*}{96} & \multirow{12}{*}{95.33} \\
\hline & & $5 \mathrm{~mm}$ & & & & \\
\hline & Defect & $7 \mathrm{~mm}$ & 58 & 2 & & \\
\hline & & $9 \mathrm{~mm}$ & & & & \\
\hline \multirow{4}{*}{2} & No defect & & 36 & 4 & \multirow{4}{*}{95} & \\
\hline & & $5 \mathrm{~mm}$ & & & & \\
\hline & Defect & $7 \mathrm{~mm}$ & 59 & 1 & & \\
\hline & & $9 \mathrm{~mm}$ & & & & \\
\hline \multirow{4}{*}{3} & No defect & & 37 & 3 & \multirow{4}{*}{95} & \\
\hline & & $5 \mathrm{~mm}$ & & & & \\
\hline & Defect & $7 \mathrm{~mm}$ & 58 & 2 & & \\
\hline & & $9 \mathrm{~mm}$ & & & & \\
\hline
\end{tabular}




\section{Table 2 (on next page)}

Test dataset recognition results 


\begin{tabular}{|c|c|c|c|c|c|c|}
\hline \multirow{2}{*}{ No. } & \multirow{2}{*}{ Class } & & \multicolumn{2}{|c|}{ Recognition result } & \multirow{2}{*}{ Accuracy $(\%)$} & \multirow{2}{*}{ Average Accuracy(\%) } \\
\hline & & & True & False & & \\
\hline \multirow{4}{*}{1} & No defect & & 19 & 1 & \multirow{4}{*}{92} & \multirow{12}{*}{91.33} \\
\hline & & $5 \mathrm{~mm}$ & & & & \\
\hline & Defect & $7 \mathrm{~mm}$ & 27 & 3 & & \\
\hline & & $9 \mathrm{~mm}$ & & & & \\
\hline \multirow{4}{*}{2} & No defect & & 18 & 2 & \multirow{4}{*}{90} & \\
\hline & & $5 \mathrm{~mm}$ & & & & \\
\hline & Defect & $7 \mathrm{~mm}$ & 27 & 3 & & \\
\hline & & $9 \mathrm{~mm}$ & & & & \\
\hline \multirow{4}{*}{3} & No defect & & 20 & 0 & \multirow{4}{*}{92} & \\
\hline & & $5 \mathrm{~mm}$ & & & & \\
\hline & Defect & $7 \mathrm{~mm}$ & 26 & 4 & & \\
\hline & & $9 \mathrm{~mm}$ & & & & \\
\hline
\end{tabular}




\section{Table 3(on next page)}

Comparison of results of 4 different methods 


\begin{tabular}{|c|c|c|c|}
\hline Model & Class & Sample number & Average $\operatorname{accuracy}(\%)$ \\
\hline $\mathrm{RBF}$ & No defect/Hole & 150 & 86 \\
\hline SVM & No defect/Hole & 150 & 87.33 \\
\hline BPNN & No defect/Hole & 150 & 86.67 \\
\hline GA-BPNN & No defect/Hole & 150 & 91.33 \\
\hline
\end{tabular}

